\title{
Lessons from Use of Continuous Glucose Monitoring Systems in Digital Healthcare
}

\author{
Hun-Sung Kim ${ }^{1,2}$, Kun-Ho Yoon ${ }^{1,2}$ \\ Departments of ${ }^{1}$ Medical Informatics, ${ }^{2}$ Endocrinology and Metabolism, College of Medicine, The Catholic University of Korea, \\ Seoul, Korea
}

We live in a digital world where a variety of wearable medical devices are available. These technologies enable us to measure our health in our daily lives. It is increasingly possible to manage our own health directly through data gathered from these wearable devices. Likewise, healthcare professionals have also been able to indirectly monitor patients' health. Healthcare professionals have accepted that digital technologies will play an increasingly important role in healthcare. Wearable technologies allow better collection of personal medical data, which healthcare professionals can use to improve the quality of healthcare provided to the public. The use of continuous glucose monitoring systems (CGMS) is the most representative and desirable case in the adoption of digital technology in healthcare. Using the case of CGMS and examining its use from the perspective of healthcare professionals, this paper discusses the necessary adjustments required in clinical practices. There is a need for various stakeholders, such as medical staff, patients, industry partners, and policy-makers, to utilize and harness the potential of digital technology.

Keywords: Blood glucose self-monitoring; Wearable electronic devices; Delivery of health care; Quality of health care

\section{INTRODUCTION}

We live in a digital healthcare era, which offers various wearable medical devices that allow us to measure our biometric and health data in our daily lives [1-3]. Digital healthcare is expected to play a pivotal role in the modern provision of patient-focused healthcare.

Traditionally, digital healthcare consisted of three components as described in Fig. 1: medical device (sensor), platform, and medical feedback [4,5]. Medical feedback, is often acknowledged as the most important of these three components. It refers to the recommendations from healthcare professionals to users, such as appropriate lifestyle guidelines. Medical feedback is informed by the data generated from the medical device.

Received: 8 April 2020, Revised: 22 June 2020, Accepted: 31 July 2020

Corresponding author: Hun-Sung Kim

Department of Medical Informatics, College of Medicine, The Catholic University of Korea, 222 Banpo-daero, Seocho-gu, Seoul 06591, Korea Tel: +82-2-2258-8262, Fax: +82-2-2258-8297, E-mail: 01 cadiz@hanmail.net
In recent years, medical devices and platforms, such as datamining engines, have played increasingly important roles in digital healthcare, influenced by rapid technological advances in information and communications technologies [6]. The ability to collect and use data to analyze patients' health and provide customized recommendations emphasizes the importance of medical devices and platforms [6]. As a result of this trend, medical feedback is increasingly data-driven and accessible to patients. This is opposed to earlier times, where medical feedback was provided only by healthcare professionals. Patients can access the information independently rather than waiting for expensive instruments or tests.

Similarly, patient-generated health data (PGHD), which refers to health data collected by patients themselves as opposed to

Copyright $\odot 2020$ Korean Endocrine Society

This is an Open Access article distributed under the terms of the Creative Commons Attribution Non-Commercial License (https://creativecommons.org/ licenses/by-nc/4.0/) which permits unrestricted non-commercial use, distribution, and reproduction in any medium, provided the original work is properly cited. 


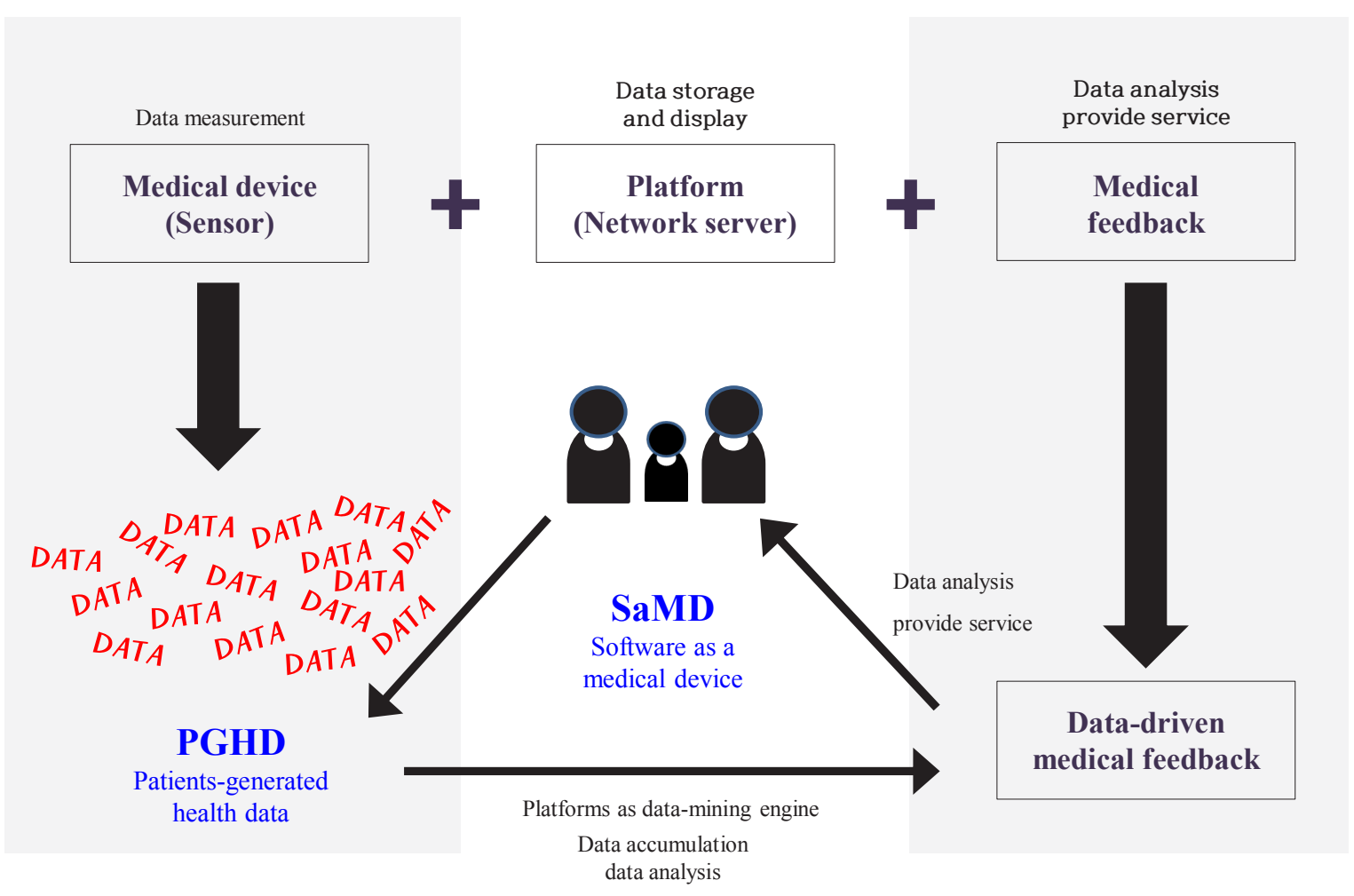

Fig. 1. Scheme of the digital healthcare system.

health data collected in hospitals, is increasingly gaining importance [7]. However, even though the medical feedback is datadriven, it is still provided by healthcare professionals. As such, the intervention of medical staff is still important in digital healthcare. However, medical staff face difficulty managing many patients, due to cost and time. Therefore, it is important to develop data-mining engines and algorithms that will assist medical staff by reducing the demands on their time and effort.

Medical data is clearly being collected in large volumes in digital healthcare. Some people believe that this focus on data generation and collection has created an exaggerated belief that data-driven digital healthcare will solve all healthcare problems. Further, a premature assumption is that several medical issues can be resolved through the analysis and use of copious amounts of data collected by wearable devices. This perception is an exaggerated claim by the medical device industry who do not fully understand the specifics of clinical practice [8]. Conversely, healthcare professionals do recognize the role that digital technologies, such as medical devices and platforms, can play in current medical work.

Within digital healthcare, the best clinically adapted example of a medical device and platform is the continuous glucose monitoring systems (CGMS). Taking the perspective of health- care professionals, we examine current uses of CGMS. We aim to understand how medical devices and platforms, as part of digital healthcare, can be applied to real clinical medical practice. In the sections below, we discuss both the meaning and limitations of this application to medical practice.

\section{CONTINUOUS GLUCOSE MONITORING SYSTEMS}

Since CGMS was approved for use by the Food and Drug Administration (FDA) in 1999, it has been used to determine the overall glucose variability in patients with diabetes mellitus [9]. This means that patients can monitor their own blood sugar trends and self-manage their blood sugar levels. Significantly, many studies indicate that the use of CGMS reduces glucose variability and the occurrence of hypoglycemia [10]. Various studies have also shown that CGMS has greatly helped in blood sugar management [10-12].

\section{CGMS as digital healthcare}

CGMS traditionally consists of a sensor to measure blood sugar levels and a transmitter that sends the measurements to a receiver in real time [13]. Previously, receivers were a separate com- 
ponent of CGMS [13]. However, recent technological advances have increasingly seen the utilization of smartphones as receivers. Blood sugar levels measured by the sensors, or scanned through linked smartphones, are communicated directly to users in real-time $[14,15]$. CGMS users can thus check their overall blood sugar values and trends on their smartphones at a glance. This ultimately helps and motivates them to actively manage their own blood sugar [14]. CGMS exemplifies the success of digital healthcare by fully exploiting its three components: the medical device; the data-mining engine platform; and the datadriven medical feedback. The use of CGMS also shifts medical feedback from being the sole domain of healthcare professionals, instead democratizing medical feedback for the user. As data is increasingly generated via the CGMS and provided directly to patients, PGHD from the wearable device is playing a more important role in healthcare. Thus, the goal of digital healthcare is to analyze the various data generated by the patient and to support patients by providing customized treatment options.

\section{Need for user training to ensure proper use}

While technological advances in digital healthcare have led to improvements in the provision of healthcare and generated a large amount of PGHD, it is unreasonable to expect users to be motivated to use a medical device. As such, it is essential to educate users in the application of digital healthcare by training users in the correct operation of medical devices such as CGMS $[6,16]$. In digital healthcare, the protagonist of healthcare is logically the users themselves. To maintain proper health in digital healthcare, users should improve their health data and health in- formation capabilities. This is a salient aspect of digital healthcare. In order to enhance their learning abilities, patients need to have deep self-knowledge and understanding so that they can better manage and interpret their health with the data generated. Patients use medical devices in diverse circumstances, with variables such as usage, machines, companies, and patients' inclination. Users will need to wear their sensors, monitor the data they receive on their devices, and learn to interpret these outputs. The education of users is thus essential to harnessing the great potential of digital healthcare.

\section{Selecting a suitable device from a variety of CGMS}

Among the currently available CGMS products, as seen in Table 1, insurance in Korea covers the purchase of Medtronic Guardian ${ }^{\mathrm{TM}}$ Connect System (Medtronic, Dublin, Ireland) [17], Dexcom's G5 ${ }^{\mathrm{TM}}$ (Dexcom, San Diego, CA, USA) [18], and Abbott's FreeStyle Libre ${ }^{\mathrm{TM}}$ (Abbott, Chicago, IL, USA) [19]. After receiving insurance benefits, the patient pays only $30 \%$ of the total device price. The sensors on Guardian Connect and G5 can be used for 7 days, and thus, four to five sensors are required per month. The two devices differ in their transmitters. Guardian Connect's transmitter can be used semi-permanently, but its parts are more expensive. It is smaller in size than earlier version, and is easy to wear. The transmitter of the G5 costs about one-third that of the Guardian Connect. However, it should not be used for more than 3 months and, consequently, requires constant replacement. Therefore, users must decide whether they want to pay more up front for a semi-permanent device, or less for a device that requires regular replacement. The sensor

Table 1. Commercially Available CGMS with Domestic Insurance Benefits $[17,18]$

\begin{tabular}{|c|c|c|c|}
\hline & Medtronic Guardian Connect System & Dexcom G5 & Abbott FreeStyle Libre \\
\hline Official dealer & $\begin{array}{l}\text { Medtronic } \\
\text { Domestic corporation }\end{array}$ & $\begin{array}{l}\text { Huons } \\
\text { Import sales }\end{array}$ & Abbott \\
\hline \multirow[t]{3}{*}{ Sensor } & Calibration at least every 12 hours & Calibration at least every 12 hours & No calibration \\
\hline & $\begin{array}{l}\text { Available for approximately } 7 \text { days } \\
\text { (4-5 sensors are required per month) }\end{array}$ & $\begin{array}{l}\text { Available for approximately } 7 \text { days } \\
\text { (4-5 sensors are required per month) }\end{array}$ & $\begin{array}{l}\text { Available for approximately } 14 \text { days } \\
\text { ( } 2 \text { required for } 28 \text { days) }\end{array}$ \\
\hline & - & - & $\begin{array}{l}\text { Relatively low price } \\
\text { Compared to other CGMS sensor }\end{array}$ \\
\hline \multirow[t]{2}{*}{ Transmitter } & Semi-permanent & $\begin{array}{l}\text { Longest recommended use period is } 3 \\
\text { months }\end{array}$ & - \\
\hline & High price & Low price & - \\
\hline Scanner & - & - & Very low price \\
\hline Etcetera & Continuous sensor glucose readings & Continuous sensor glucose readings & $\begin{array}{l}\text { Interlocking with an insulin pump is also } \\
\text { impossible }\end{array}$ \\
\hline
\end{tabular}


of the recently released FreeStyle Libre can measure blood glucose continuously for up to 14 days. The FreeStyle Libre is priced competitively compared to other CGMS because data is sent to a smartphone every time it is scanned. However, the FreeStyle Libre does not continuously transmit blood sugar data. Thus, it is unable to monitor and report on predicted hypoglycemia. It also cannot integrate an artificial pancreas with an insulin pump. The CGMS purchase decision should always be made in consultation with medical providers, taking into consideration the purpose of use, cost, and personal situation, among others. Also, clinical results may differ depending on how these digital devices are used [20].

\section{Understanding the limitations of CGMS}

There are several limitations to the use of CGMS. First, CGMS users should be aware of the physiological lag time of the CGMS they use. There is a gap of 1 to 5 minutes between the blood sugar reading and the CGMS measurements displayed to the user [21]. Thus, the blood sugar values are not shown in perfect real-time. This is why users should not be absolutely dependent on these measurements. Consequently, we have suggested a predictive alarm based on data trends for hypoglycemia.

Second, it is necessary to calibrate the blood glucose level measured by the CGMS with self-measured blood glucose levels. A common misunderstanding by many users is that when they wear the CGMS, they do not need to measure their own blood sugar level at all. For instance, the FreeStyle Libre is precalibrated for blood sugar values and users can use it for 2 weeks without individual calibration. While some CGMS do not require calibration, others require recalibration twice daily [22]. This, however, is an improvement, given that older CGMS used to require calibration four times a day $[13,16]$. While CGMS have significantly improved in terms of accuracy, performance, and hardware (such as sensor size, price, waterproofing, battery life, weight), it is also important to note that the measurable blood sugar range is limited [23-25].

The dependency on CGMS is also its limitation. High dependency on a medical device leads to more serious consequences in the case of errors. Consequently, the accuracy of the measured value cannot be overstated when using CGMS [26,27]. Moreover, this limitation may not be resolved by simply emphasizing the accuracy of measurement, which is one of the basic requirements of medical devices. Thus, CGMS should emphasize the provision of customized content based on the stored PGHD. For instance, the PGHD can enable CGMS to predict patients' indicative values for normoglycemia. This provides
CGMS the capability to estimate when these normative values are exceeded. Predictive, system-generated text notifications for hypo- or hyper-glycemia can then be sent to alert the user $[13,28]$. In addition, simultaneous alerts can be targeted at the patient's caregiver.

\section{NEED FOR ACTIVE PARTICIPATION OF MEDICAL PROFESSIONALS}

The role of medical professionals in digital healthcare is also important $[8,29]$. While medical feedback is increasingly datadriven, the active participation of medical professionals can complement and improve the provision of digital healthcare. One way to do this is for medical professionals to leverage PGHD to educate patients and manage their ailments. Medical professionals can use PGHD to create a proper treatment plan and guide patients directly. This is because patients might not have the necessary expertise to understand PGHD if they use it themselves [13]. Research has shown that satisfaction with medical devices and platforms is positively correlated to healthcare outcomes: when one is low, the other is also not desirable [30]. Medical professionals should recognize that CGMS is also a medical device. The purpose of CGMS is not to make medical decisions, but to support medical professionals as they make decisions in the best interests of their patients [29]. While patients can now manage their own health through CGMS, they still need professional medical advice when things are beyond their control. The use of CGMS by healthcare professionals to complement patient self-care and management is the ideal combination of technology and medical care and exemplifies the potential that digital healthcare can play in the world today.

\section{THE FUTURE OF PERSONALIZED AND CUSTOMIZED HEALTH CARE}

In the digital healthcare era, various wearable devices aim to ultimately provide personalized and customized services for users. Traditional artificial intelligence (AI) was created using prediction models founded on rule-based algorithms [31]. However, these algorithms are inadequate for individualized and customized health management. In contrast, the AI incorporated into CGMS utilizes a combination of data uploaded by both users and algorithms. As such, it offers more individualized customizations than traditional AI. In the future, data accumulated by users, such as sleeping and eating patterns, and exercise information, will be used along with blood glucose data to predict 
individual blood sugar patterns [8]. Various trials on predicting individual blood sugar levels are underway, and commercial versions are being prepared [32-34]. Similarly, AI will be able to predict differing, individualized blood sugar patterns, even when the users maintain the same diet and exercise levels.

In the near future, with the advancement of digital technologies, an artificial pancreas, combined with CGMS and insulin pumps, is expected to be commercialized for safe use, without concern about hypoglycemia $[35,36]$. To the best of our knowledge, there is currently no research in Korea on the monitoring of an artificial pancreas with an independent algorithm. As such, extensive attention by medical staff is required. Advances in IT will promote the provision of personalized medical services, while enabling more accurate and efficient responses to an individual patient's blood sugar status. Additionally, the type and amount of data collected will increase, and more refined data will improve the quality of the results inferred from algorithms [8]. Importantly, for useful blood glucose data to be created, devices need to be continuously worn. As indicated in one study, it is practically impossible to obtain clinical benefits if a user does not have a high level of adherence $(\geq 70 \%)$ [37]. In the future, the aim of these learning algorithms in CGMS will be to use information to alert the user to hypoglycemia and hyperglycemia. This will enable CGMS to independently discover the individualized baseline of the patient's blood sugar pattern.

\section{NEED FOR EXPERT GROUPS AND DIGITAL HEALTH COORDINATING CENTER}

We cannot overlook the importance of policy support in harnessing the potential of digital healthcare. A significant amount of training time would need to be invested in first-time users of CGMS, rather than the 5 to 10 minutes of training that is currently offered [16]. It is essential that users are trained in recognizing and managing their blood sugar levels and how to deal with fluctuations, rather than receiving only simple training in using the CGMS. Additionally, we recommend that patients attend refresher training every time they visit the hospital. In summary, a group of CGMS experts is needed, including doctors and nurses, and a system needs to be implemented to develop

\section{Digital Health Coordinating Center}

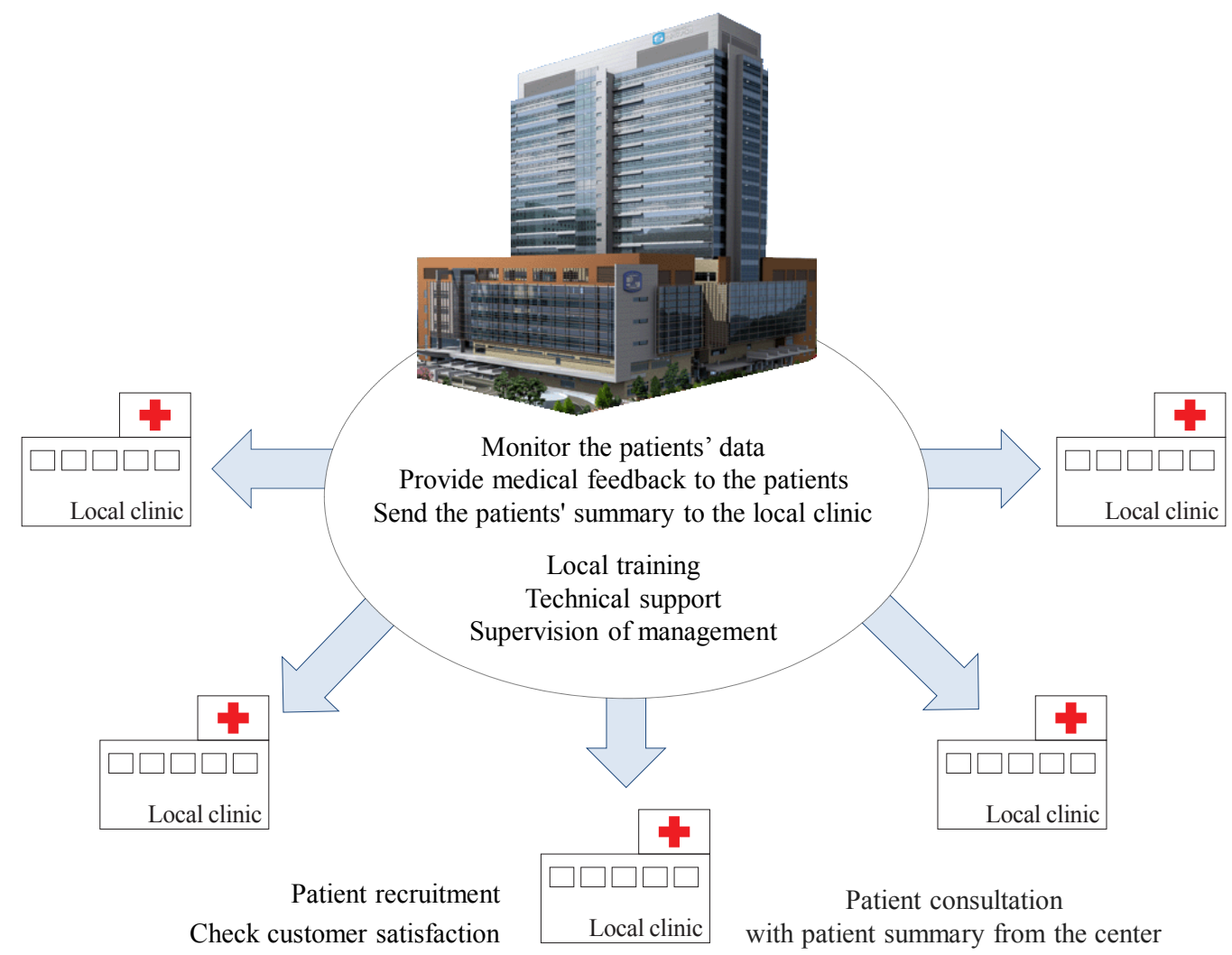

Fig. 2. Schema of regional-based Digital Health Coordinating Center [13,26]. 
these experts [13].

Consequently, we recognize that activating CGMS to harness the potential of digital healthcare in local clinics is not an easy task in Korea. We propose a regional-based Digital Health Coordinating Center (DHCC) (Fig. 2) as the control and coordinating center to support local clinics $[13,26,38]$. When a physician prescribes CGMS at a local clinic, the DHCC will be responsible for educating the patient regarding its use and necessary precautions. Users will visit the DHCC once for training, and thereafter will continue to visit their local clinic and primary care provider. PGHD generated and stored through the CGMS will be transmitted to the DHCC, and medical professionals at the DHCC should monitor these measurements. The DHCC will then provide summarized information and health feedback from the PGHD to the patients through the local clinic. This will allow medical professionals at local clinics to focus solely on treating the patients. This will also reduce the burden on local clinics and strengthen the DHCC's expertise in digital healthcare. However, areas such as health insurance, personal information protection, responsibility for medical accidents, and provision of healthcare incentives need to be resolved before the DHCC is set up.

\section{NON-INVASIVE WEARABLE TECHNOLOGY IN ENDOCRINOLOGY FIELD}

Digital healthcare in endocrinology is mostly concentrated on diabetes mellitus. In addition to CGMS, various studies have investigated the measurement of blood glucose levels through other non-invasive methods, such as contact lens [39-42], sweat [43], breathing [44], or tattoo-based blood glucose measurement [45]. These studies are progressing towards commercialization. The common goal of these non-invasive methods of measuring blood glucose is to relieve patients of the burden of puncturing their finger with a needle. Likewise, the focus of digital healthcare wearable devices (sensors) is to minimize this burden. However, many challenges need to be resolved before clinical application is possible, such as size, price of the sensor and transmitter, and various technological barriers. In addition, medical devices such as pedometers [46] and exercise trackers [47], which manage the active lifestyle of diabetics, continue to receive attention.

\section{CONCLUSIONS}

In order to harness the potential of CGMS in digital healthcare, we need to strengthen the medical capabilities of digital healthcare and pay greater attention to the various stakeholders in the ecosystem, such as medical professionals, patient-users, and policy-makers. The eventual aim for CGMS in digital healthcare is to manage and improve patients' health. To achieve this goal, medical professionals should encourage and educate patients on proper self-care. Medical staff should actively use technology in healthcare to improve the quality of medical care. To achieve this, they must have an unbiased interest in learning about and understanding digital devices and platforms that exist.

A critical feature of CGMS and other forms of digital healthcare should be to on real-time monitoring so as to provide customized solutions. To advance CGMS as a digital healthcare solution of the future, we need to focus on improving the collection, investigation, and analysis of personal data. We need to define which data to collect or discard, and focus on using data to produce clinically useful results. In addition, by accessing information stored on the patient's device, we can move beyond the limitations of the fixed variables of age and sex. This will enable us to accurately predict the user's condition based on variables, such as season, weather, wake-up time, and activity time. AI may be the enabler of proactive, customized treatments before users are aware they need it. While initial improvements in $\mathrm{AI}$ are not expected to solve all problems, the need for further research using CGMS as the starting point, is compelling.

\section{CONFLICTS OF INTEREST}

No potential conflict of interest relevant to this article was reported.

\section{ACKNOWLEDGMENTS}

This work was supported by the Technology Development Program (S2726209) funded by the Ministry of SMEs and Startups (MSS, Korea).

\section{ORCID}

Hun-Sung Kim https://orcid.org/0000-0002-7002-7300

\section{REFERENCES}

1. Karinharju KS, Boughey AM, Tweedy SM, Clanchy KM, Trost SG, Gomersall SR. Validity of the Apple Watch ${ }^{\circledR}$ for monitoring push counts in people using manual wheelchairs. 
J Spinal Cord Med 2019:1-9.

2. Hernando D, Roca S, Sancho J, Alesanco A, Bailon R. Validation of the Apple watch for heart rate variability measurements during relax and mental stress in healthy subjects. Sensors (Basel) 2018;18:2619.

3. Raja JM, Elsakr C, Roman S, Cave B, Pour-Ghaz I, Nanda A, et al. Apple watch, wearables, and heart rhythm: where do we stand? Ann Transl Med 2019;7:417.

4. Kim HS, Cho JH, Yoon KH. New directions in chronic disease management. Endocrinol Metab (Seoul) 2015;30:15966.

5. Kim HS, Kim H, Lee S, Lee KH, Kim JH. Current clinical status of telehealth in Korea: categories, scientific basis, and obstacles. Healthc Inform Res 2015;21:244-50.

6. Lee J, Kim HS, KIM DJ. Recent technology-driven advancements in cardiovascular disease prevention. Cardiovasc Prev Pharmacother 2019;1:43-9.

7. Nittas V, Lun P, Ehrler F, Puhan MA, Mutsch M. Electronic patient-generated health data to facilitate disease prevention and health promotion: scoping review. J Med Internet Res 2019;21:e13320.

8. Kim HS, Kim DJ, Yoon KH. Medical big data is not yet available: why we need realism rather than exaggeration. Endocrinol Metab (Seoul) 2019;34:349-54.

9. Mastrototaro J. The MiniMed continuous glucose monitoring system (CGMS). J Pediatr Endocrinol Metab 1999;12 Suppl 3:751-8.

10. Korean Diabetes Association. Treatment guideline for diabetes 2015. Seoul: Korean Diabetes Association; 2015.

11. Chetty VT, Almulla A, Odueyungbo A, Thabane L. The effect of continuous subcutaneous glucose monitoring (CGMS) versus intermittent whole blood finger-stick glucose monitoring (SBGM) on hemoglobin A1c (HBA1c) levels in type I diabetic patients: a systematic review. Diabetes Res Clin Pract 2008;81:79-87.

12. Wei Q, Sun Z, Yang Y, Yu H, Ding H, Wang S. Effect of a CGMS and SMBG on maternal and neonatal outcomes in gestational diabetes mellitus: a randomized controlled trial. Sci Rep 2016;6:19920.

13. Kim HS, Shin JA, Chang JS, Cho JH, Son HY, Yoon KH. Continuous glucose monitoring: current clinical use. Diabetes Metab Res Rev 2012;28 Suppl 2:73-8.

14. Anderson SM, Dassau E, Raghinaru D, Lum J, Brown SA, Pinsker JE, et al. The international diabetes closed-loop study: testing artificial pancreas component interoperability. Diabetes Technol Ther 2019;21:73-80.
15. Fernandez-Carames TM, Froiz-Miguez I, Blanco-Novoa O, Fraga-Lamas P. Enabling the internet of mobile crowdsourcing health things: a mobile fog computing, blockchain and IOT based continuous glucose monitoring system for diabetes mellitus research and care. Sensors (Basel) 2019;19: 3319.

16. Jung SH, Kim JW, Kang IK, Park CY, Kim YS, Woo JT, et al. Continuous glucose monitoring is needed to detect unrecognized hypoglycemic event in diabetic patients with stroke. J Korean Diabetes 2002;3:140-51.

17. Medtronic. The guardian connect CGM [Internet]. Dublin: Medtronic; 2018 [cited 2020 Aug 10]. Available from: https:// www.medtronicdiabetes.com/products/guardian-connectcontinuous-glucose-monitoring-system.

18. Dexcom Inc. The Dexcom G5® Mobile CGM System [Internet]. San Diego: Dexcom; 2019 [cited 2020 Aug 10]. Available from: https://www.dexcom.com/g5-mobile-cgm.

19. Abbott. FreeStyle Libre [Internet]. Chicago: Abbott; 2020 [cited 2020 Aug 10]. Available from: https://www.freestylelibre. co.uk/libre/.

20. Kim HS, Choi W, Baek EK, Kim YA, Yang SJ, Choi IY, et al. Efficacy of the smartphone-based glucose management application stratified by user satisfaction. Diabetes Metab J 2014; 38:204-10.

21. Basu A, Dube S, Veettil S, Slama M, Kudva YC, Peyser T, et al. Time lag of glucose from intravascular to interstitial compartment in type 1 diabetes. J Diabetes Sci Technol 2015;9: 63-8.

22. Bergenstal RM. Continuous glucose monitoring: transforming diabetes management step by step. Lancet 2018;391: 1334-6.

23. Heinemann L. Continuous glucose monitoring (CGM) or blood glucose monitoring (BGM): interactions and implications. J Diabetes Sci Technol 2018;12:873-9.

24. Knebel T, Neumiller JJ. Medtronic MiniMed 670G hybrid closed-loop system. Clin Diabetes 2019;37:94-5.

25. Mazze RS. Acceptance of FGM or CGM in clinical decision-making and patient preference: where do we go from here? Diabetes Technol Ther 2017;19:142-4.

26. Kim HS, Lee KH, Kim H, Kim JH. Using mobile phones in healthcare management for the elderly. Maturitas 2014;79: 381-8.

27. Kim HS, Hwang Y, Lee JH, Oh HY, Kim YJ, Kwon HY, et al. Future prospects of health management systems using cellular phones. Telemed J E Health 2014;20:544-51.

28. Abraham SB, Arunachalam S, Zhong A, Agrawal P, Cohen 
O, McMahon CM. Improved real-world glycemic control with continuous glucose monitoring system predictive alerts. J Diabetes Sci Technol 2019 Jul 4 [Epub]. https://doi. org/10.1177/1932296819859334.

29. Kim HS. Decision-making in artificial intelligence: is it always correct? J Korean Med Sci 2020;35:e1.

30. Kim HS, Yang SJ, Jeong YJ, Kim YE, Hong SW, Cho JH. Satisfaction survey on information technology-based glucose monitoring system targeting diabetes mellitus in private local clinics in Korea. Diabetes Metab J 2017;41:213-22.

31. Lim I, Walkup RK, Vannier MW. Rule based artificial intelligence expert system for determination of upper extremity impairment rating. Comput Methods Programs Biomed 1993; 39:203-11.

32. Ratheau L, Jeandidier N, Moreau F, Sigrist S, Pinget M. How technology has changed diabetes management and what it has failed to achieve. Diabetes Metab 2011;37 Suppl 4:S57-64.

33. Reymann MP, Dorschky E, Groh BH, Martindale C, Blank P, Eskofier BM. Blood glucose level prediction based on support vector regression using mobile platforms. Conf Proc IEEE Eng Med Biol Soc 2016;2016:2990-3.

34. Seo W, Lee YB, Lee S, Jin SM, Park SM. A machine-learning approach to predict postprandial hypoglycemia. BMC Med Inform Decis Mak 2019;19:210.

35. Weisman A, Bai JW, Cardinez M, Kramer CK, Perkins BA. Effect of artificial pancreas systems on glycaemic control in patients with type 1 diabetes: a systematic review and metaanalysis of outpatient randomised controlled trials. Lancet Diabetes Endocrinol 2017;5:501-12.

36. Boughton CK, Hovorka R. Is an artificial pancreas (closedloop system) for type 1 diabetes effective? Diabet Med 2019; 36:279-86.

37. Yu S, Varughese B, Li Z, Kushner PR. Healthcare resource waste associated with patient nonadherence and early discontinuation of traditional continuous glucose monitoring in real-world settings: a multicountry analysis. Diabetes Technol Ther 2018;20:420-7.

38. Kim HS, Sun C, Yang SJ, Sun L, Li F, Choi IY, et al. Randomized, open-label, parallel group study to evaluate the effect of internet-based glucose management system on subjects with diabetes in China. Telemed J E Health 2016; 22:666-74.

39. Lin YR, Hung CC, Chiu HY, Chang BH, Li BR, Cheng SJ, et al. Noninvasive glucose monitoring with a contact lens and smartphone. Sensors (Basel) 2018;18:3208.

40. Ascaso FJ, Huerva V. Noninvasive continuous monitoring of tear glucose using glucose-sensing contact lenses. Optom Vis Sci 2016;93:426-34.

41. Zhang J, Hodge W, Hutnick C, Wang X. Noninvasive diagnostic devices for diabetes through measuring tear glucose. J Diabetes Sci Technol 2011;5:166-72.

42. O’Donnell C, Efron N. Diabetes and contact lens wear. Clin Exp Optom 2012;95:328-37.

43. Lee H, Song C, Hong YS, Kim MS, Cho HR, Kang T, et al. Wearable/disposable sweat-based glucose monitoring device with multistage transdermal drug delivery module. Sci Adv 2017;3:e1601314.

44. Tankasala D, Linnes JC. Noninvasive glucose detection in exhaled breath condensate. Transl Res 2019;213:1-22.

45. Bandodkar AJ, Jia W, Yardimci C, Wang X, Ramirez J, Wang J. Tattoo-based noninvasive glucose monitoring: a proof-of-concept study. Anal Chem 2015;87:394-8.

46. Rossen J, Yngve A, Hagstromer M, Brismar K, Ainsworth $\mathrm{BE}$, Iskull $\mathrm{C}$, et al. Physical activity promotion in the primary care setting in pre- and type 2 diabetes: the Sophia step study, an RCT. BMC Public Health 2015;15:647.

47. Groat D, Kwon HJ, Grando MA, Cook CB, Thompson B. Comparing real-time self-tracking and device-recorded exercise data in subjects with type 1 diabetes. Appl Clin Inform 2018;9:919-26. 